\title{
1. A marginal abatement cost curve for material efficiency accounting for uncertainty \author{
Cullen $^{\mathrm{a}}$, Julian M. Allwood ${ }^{\mathrm{a}}$ \\ ${ }^{a}$ Department of Engineering, University of Cambridge, Trumpington Street, Cambridge CB2 1PZ, UK
} \\ Cyrille F. Dunant ${ }^{\mathrm{a}, *}$, Alexandra C. H. Skelton ${ }^{\mathrm{a}}$, Michał P. Drewniok ${ }^{\mathrm{a}}$, Jonathan M.
}

6 Abstract

Comparing costs of measures to mitigate greenhouse gas is challenging as there are many competing notions of costs, and uncertainties associated with cost estimates. In addition, there are many different types of mitigation measures, from supply-side investment solutions to demand-side efficiency improvements, which may interact, risking doublecounting of abatement potentials. This paper presents a novel, transparent methodology for building a marginal abatement cost curve that allows abatement costs and potentials to be compared. This curve improves over existing methods as it allows for abatement measures to be pursued in parallel, takes into account the interplay between abatement measures and captures data on cost uncertainty. The method is applied to build the first bottom-up marginal abatement cost curve for greater material efficiency steel use in the UK. This curve is demonstrated via four material efficiency measures which do not require large changes in final uses of products: reusing steel beams in construction, specifying optimal lightweight beams in construction, choosing smaller cars and specifying high strength steel car bodies. The results show that these strategies could reduce uK steel demand and associated global emissions by approximately $12 \% .17 \%$ of this potential would be viable at the Department for Business, Energy \& Industrial Strategy (BEIS) 2030 carbon price for policy appraisal $\left(79 £ / \mathrm{tco}_{2}\right)$ taking into account emissions savings associated with steel demand only. Once use-phase emissions savings are taken into account this share increases to $60 \%$. These results can be traced directly back to

\footnotetext{
${ }^{*}$ Corresponding author
} 
underlying assumptions regarding costs and emissions allocations.

Keywords: MAcc, Material efficiency, Policy guidance

8 1. Introduction

Marginal abatement cost curves (MAccs) seek to convert the cost of different greenhouse gas (GhG) emissions abatement measures into comparable, $£ / \mathrm{tcO}_{2}$, units. This is helpful for prioritising measures, but the application of the method has been heavily criticised. Concerns, expressed by Kesicki and Strachan (2011-12) and Ekins et al. (2011) amongst others, include the lack of transparency regarding underlying assumptions (in particular in high profile work by McKinsey (2009)), the failure to account for the interaction between strategies (which can lead to double-counting in reduction potentials), and the limited representation of uncertainty. Despite their limitations, MACCs, as for example Figure 1, continue to be used to inform the policy community of the cost of disparate emissions abatement options. For example Eory et al. (2015) use a MACC to assess the potential contribution of measures in the agricultural sector to the UK $5^{\text {th }}$ Carbon Budget period and the uk government continues to estimate annual traded carbon values for the purpose of policy appraisal (DECC 2009, Department for Business, Energy \& Industrial Strategy (BEIS) 2018 ). Given the emphasis on integrated assessment models in the IPCC process (Clarke and Jiang, 2014), measures that cannot be readily incorporated into these models in the form of MAccs may not be given due attention in the analysis of mitigation pathways to meet the international climate commitments set by the Paris Accord.

In this context, the challenge is not only to improve MAcc transparency and methods but also to ensure that MAccs can be used to describe the full gamut of emissions options available. Strategies involving greater efficiency in the use of energy-intensive bulk materials (such as steel) have been shown to offer significant mitigation potential but remain under-represented in MAccs. As explained by Allwood et al. (2011), steel accounts for a quarter of global industrial carbon emissions and there is ample opportunity to improve 
the efficiency with which the metal is used, in particular in the construction sector (where approximately half of the steel in office buildings is surplus to requirements (Moynihan and Allwood, 2014; Dunant et al., 2018a) and the automotive sector (where $40 \%$ of steel

36 is scrapped along supply chains (Horton and Allwood, 2017)). Although model derived cost curves that explore the relationship between resource efficiency and aggregate GDP exist (Distelkamp and Meyer, 2014), to our knowledge there are no studies that draw on bottom-up engineering cost data to explore the implied marginal cost of abatement of

$40 \quad$ specific material efficiency measures.

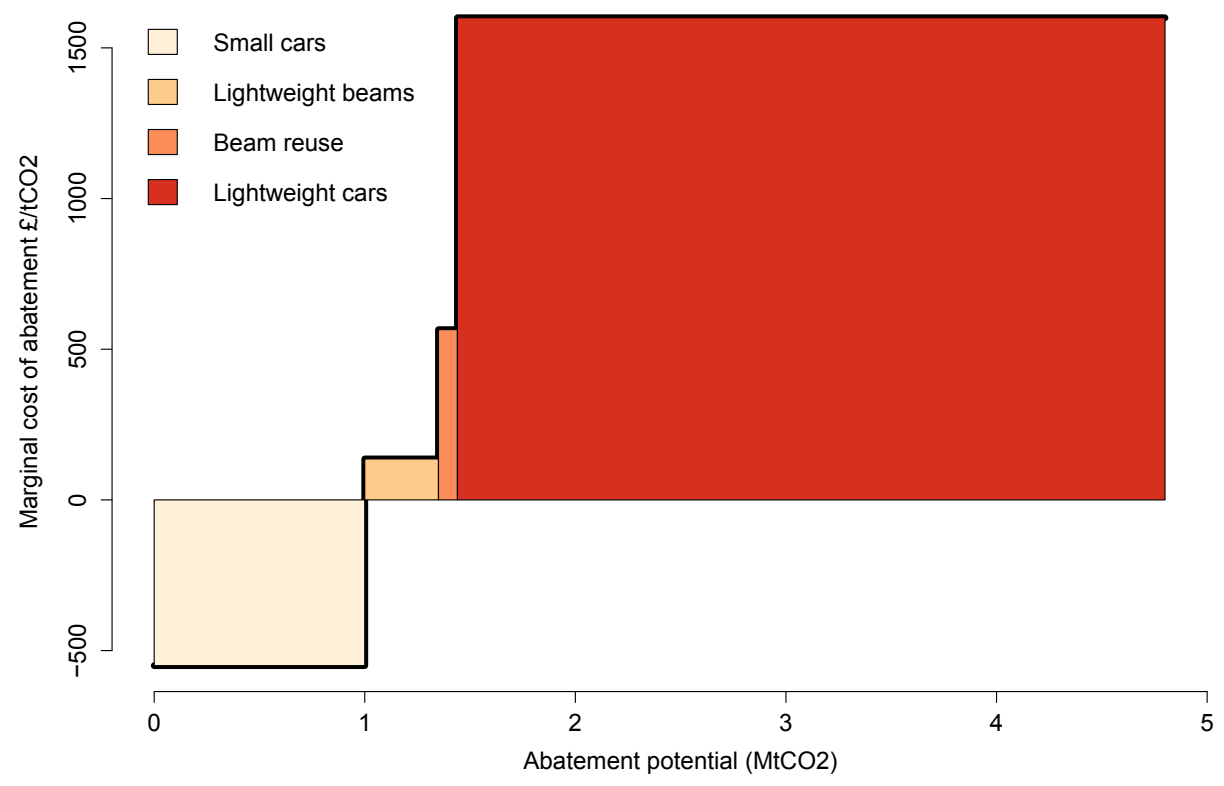

Figure 1: Material efficiency measures on a traditional MAcc

${ }_{41}$ In light of these findings, this paper seeks both to improve the MAcc methodology and

42 to extend the scope of maccs to incorporate material efficiency measures. By proposing

43 a new MACC methodology and applying it to assess the marginal cost of abatement of

44 four strategies that improve material efficiency in the the use of steel in the UK, we hope

45 to answer the following research questions:

- What information must be provided in order to be transparent about the assump- 


\section{Method}

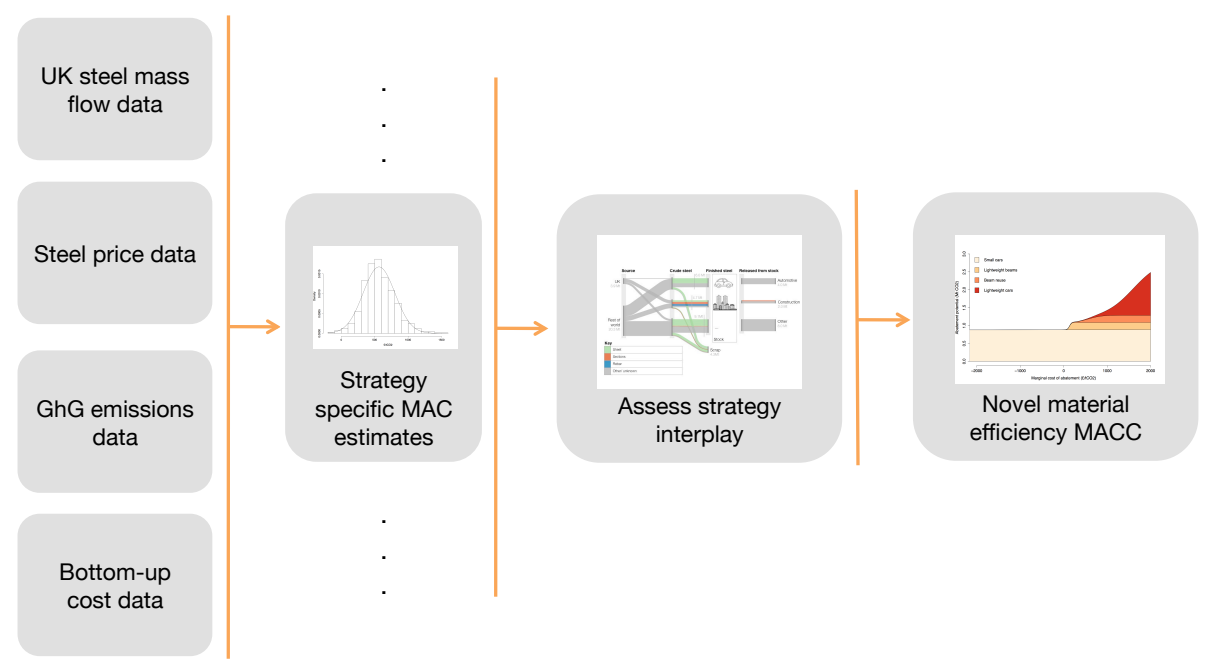

Figure 2: Proposed material efficiency MACC method tween strategies have on the material efficiency MAcc?

- How does the marginal cost of material efficiency strategies compare to the reported cost of other abatement options?
- What effect does incorporating uncertainty and accounting for the interplay be-

Figure 2 gives an overview of the proposed method. The following steps are used to draw the curve.

1. For each material efficiency measure, we collect bottom-up data on measure specific costs.

2. We verify whether these measures do not affect the use patterns of the final products, and their effects are not dependent on social factors.

3. We combine these measure-specific cost assumptions with cross-measure assumptions. 
4. To assess which measures interact with each other we map the material efficiency measures to be included in the MACC onto a Sankey diagramme and identify measures that relate to inter-dependent flows.

5. To account for the interactions between strategies, we express the potential for each strategy in terms of common parameters. We then estimate the degree to which measure $a$ can be applied, for a given application of measure $b\left(\eta_{a \mid b}\right)$ and use this to restrict the emissions abatement potential.

6. Finally, to build the MACC we combine CDF cost curves for each of the strategies, taking into account the interplay between the strategies.

\subsection{Estimators, variability and uncertainty}

We lay out here how particular difficulties or ambiguities can be resolved, depending on the nature of the data.

Choice of estimators. To account for both the uncertainty in the data and its variability (price fluctuations over time, aggregation of technological variants), the cost and carbon data should be translated to probability distributions. The choice of the distributions can be done after inspection of the data, to provide the best fit in each case and have valid bounds. Provided a sufficiently large dataset, it can be preferable to use smoothed density estimators, which would exploit the real distributions in price and carbon, but for the purpose of this methodological paper, using simple univariate distributions is sufficient.

Distinguishing between different types of variability and uncertainty. A single MAcc can only be drawn for a particular set of contextual parameters e.g. the underlying price of labour and material, the cost of capital and the state of technology. Given uncertainty over these contextual parameters, they must be fixed in order to draw a particular curve that describes a particular state of the world. Even when these parameters are fixed we would expect the cost of a particular strategy to vary to reflect any economies and 
diseconomies of scale as the strategy is exploited. We therefore distinguish between the "variability" in strategy costs (to be taken into account in a particular MACC) and "uncertainty" in cross-strategy contextual parameters (to be taken into account across different curves). This distinction is particularly important for dealing consistently with interplay between strategies. If uncertain cross-strategy parameters are not fixed across strategies, there is a risk that incompatible facts are assumed to happen simultaneously e.g. that a high steel price application of one strategy is assumed to be exploited at the same time as a low steel price application of another strategy.

\subsection{Identifying the policy impetus within an economic paradigm}

The marginal cost of abatement depends in part on the policy impetus assumed. This could be an existing policy mechanism (e.g. the EU ETS — the European Union Emissions Trading Scheme), a theoretical construct (e.g. a universal carbon price on all emissions, globally) or a new form of regulation (e.g. specifying a maximum weight standard for car bodies). The chosen policy impetus has implications for the types of costs taken into account and for the way that these costs are passed on along supply chains. For example, we assume that the smaller car strategy is motivated by a regulation that sets a maximum weight for each vehicle type. As a result we only estimate the change in the price of the vehicle. The disutility due to constraining the choice of the consumer remains hidden. If instead this strategy were to be motivated by a carbon price this disutility would have to be taken into account and consumers would have to be compensated to overcome this loss in welfare.

If the strategy were to be motivated by a particular carbon pricing scheme, e.g. the EU ETS, then constraints on the way that this scheme is implemented (e.g. geographic jurisdiction and sectoral coverage) and the impact of related policies (e.g. the compensation for indirect carbon costs in heavy industries) should be taken into account in calculating the carbon price required to instigate change. Skelton and Allwood have shown that cost-pass-through of material efficiency in the EU ETs is inefficient, meaning 
that simply converting the cost of implementing downstream material efficiency measures into a carbon price (referred to as the shadow price of carbon) will under-estimate the costs of incentivising measures through this particular mechanism.

As explained by Skelton and Allwood, the rate of cost-pass-through depends in part on the economic paradigm assumed: firms operating under perfect competition have no choice put to fully pass on their costs whereas firms with some market power face a strategic decision over whether to absorb cost increases in profits or pass them on to their customers. Economic paradigm also has a large bearing on the way that costs are interpreted. For example, in estimating the cost choosing the lightest beams permitted by building codes we assume that designers are rational in their current decisions and consequently that the additional cost paid for steel that is not required in buildings must equal the benefit of excess steel (e.g. economies of scale in purchasing and flexibility in the face of possible future design changes). Thus the "cost" can be interpreted as a "benefit".

\section{Estimating the material efficiency MACC - steel use in the UK}

To demonstrate the usefulness of the proposed approach, we apply it to a specific case not well captured by more traditional MAcc: steel use in th UK. We focus on four specific material efficiency measures for our exemplar curve: reuse of steel sections in construction, choosing minimal steel beams, lightweight vehicles and choosing smaller cars. The measures were chosen to represent the two key steel end-use sectors in the uk: the automotive and construction industry. We combine measure-specific cost assumptions with cross-measure assumptions regarding steel prices, scrap prices and the emissions intensity of steel production to yield strategy specific marginal abatement cost (MAC) distributions.

The process of populating the curve for four material efficiency measures illustrates the types of assumptions that have to be made in order to build the material efficiency MAcc. In the discussion section we draw together these assumptions to build a possible 
standard for documenting MACC assumption, explore the value of the novel MACC method proposed and compare the resulting material efficiency abatement cost estimates to other GhG emissions abatement options.

\subsection{Overview of strategy specific cost estimates}

Table 1 gives a summary of the parameters used to estimate the marginal cost of abatement for each of these measures. The resulting marginal abatement cost distributions are given in Table 2. Full details of the assumptions and data sources that underpin these cost estimates are provided in the Supplementary Information and briefly summarised below.

Table 1: MAC cost assumptions. The distributions used are Uniform, U(min, max), Triangular, Tri(min, max, peak), Normal, $\mathrm{N}(\mu, \sigma)$, and Log-Normal $\mathrm{LN}(\mu, \sigma)$.

\begin{tabular}{lllll}
\hline Strategy & Cost Measure & Ab. & Units & Estimate \\
\hline Beam reuse & Deconstruction & $c_{d}$ & $\mathfrak{f} / \mathrm{t}$ fin. steel & $\sim \mathrm{U}(70,147)$ \\
& Reconditioning & $c_{r}$ & $\mathfrak{f} / \mathrm{t}$ fin. steel & $\sim \mathrm{U}(247,376)$ \\
& Transportation & $c_{t}$ & $\mathfrak{t} / \mathrm{t}$ fin. steel & $\sim \mathrm{U}(44,50)$ \\
& Location premia & - & Excluded & - \\
& CE marking costs & - & Excluded & - \\
\multirow{5}{*}{ Lightweight beam } & Scheduling costs & - & Excluded & - \\
& Cost of excess steel & $\beta$ & $\%$ of $p_{s}$ & $\sim \operatorname{Tri}(18,81,51)$ \\
& Rationalisation benefit & - & Inferred & - \\
Hss car body & Insurance change design & - & Inferred & - \\
& Lightweighting cost & $c_{l}$ & $\mathfrak{f} / \mathrm{t}$ fin. steel & $\sim \mathrm{U}(2490,4270)$ \\
& Use phase mass cost saving & $p_{u}$ & $\mathfrak{f} / \mathrm{t}$ steel saved & $\sim \mathrm{U}(-1500,-7000)$ \\
& Powertrain cost savings & - & Excluded & - \\
All & Vehicle price & $p_{v}$ & $\mathfrak{f} / \mathrm{t}$ steel & $\sim \operatorname{Tri}(-240 \mathrm{~K}, 52 \mathrm{~K}, 43 \mathrm{~K})$ \\
& Car body price & $\gamma$ & $\%$ of $p_{v}$ & $\sim \operatorname{Tri}(1,6,3)$ \\
& Use phase mass cost saving & $p_{u}$ & $\mathfrak{f} / \mathrm{t}$ steel saved & $\sim \mathrm{U}(-1500,-7000)$ \\
& Steel section price & $p_{s}$ & $\mathfrak{f} / \mathrm{t}$ fin. steel & $\sim \mathrm{LN}(6.1,0.2)$ \\
& Steel scrap price & $\alpha$ & $\%$ of $p_{s}$ & $\sim \mathrm{N}(41,9)$ \\
& Emissions BF-BoF & $m_{1}$ & $\mathrm{tCO}_{2} / t$ cr. steel & $\sim \mathrm{N}(2.25,0.48)$ \\
& Emissions EAF & $m_{2}$ & $\mathrm{tCO} 2 / t$ cr. steel & $\sim \mathrm{N}(0.41,0.07)$ \\
& Car use phase emissions & $m_{u}$ & $\mathrm{tCO}_{2} / t$ crude steel & $\sim \mathrm{U}(0.52,0.64)$ \\
\hline
\end{tabular}

Beam reuse. The physical properties of steel beams do not deteriorate over time unless beams are exposed to extreme conditions such as fire. When a building is no longer required it can be deconstructed to extract the beams to be reconditioned for reuse in new 
Table 2: Measure specific cost distributions. The detail of the formulae and the values for the various coefficients are found in the sI.

\begin{tabular}{lllll}
\hline Measure & Sector & Emissions savings & MAcc distribution & MAcc equation \\
\hline Beam reuse & Construction & Embodied & $\sim \mathrm{N}(565,238)$ & $\frac{\left(p_{s} \alpha+c_{d}+c_{r}+c_{t}\right)-p_{s}}{m_{2}}$ \\
Lightest beam & Construction & Embodied & $\sim \mathrm{N}(136,54)$ & $\frac{\beta \cdot p_{s}}{\left(0.74 \cdot m_{1}+0.26 \cdot m_{2}\right)}$ \\
HSs car body & Automotive & Embodied & $\sim \mathrm{N}(1600,472)$ & $\frac{c_{l}}{m_{1}}$ \\
& Automotive & Embodied \& use-phase & $\sim \mathrm{N}(-338,598)$ & $\frac{c_{l}+c_{u}}{m_{1}+m_{u}}$ \\
Smaller car & Automotive & Embodied & $\sim \mathrm{N}(-550,864)$ & $\frac{\gamma c_{v}}{m_{1}}$ \\
& Automotive & Embodied \& use-phase & $\sim \mathrm{N}(-2135,1150)$ & $\frac{\gamma c_{v}+c_{u}}{m_{1}+m_{u}}$ \\
\hline
\end{tabular}

buildings. Only a small fraction of beams are currently reused in the UK. A survey of UK demolition contractors by Sansom and Avery (2014-06) revealed that $5 \%$ of beams were reused in 2012. Instead the vast majority of buildings are demolished, damaging the beams, meaning that they have to be recycled rather than reused. This was the end-of-life route for $93 \%$ of beams in the uk in 2012 (Sansom and Avery, 2014-06). The deconstruction premium, reconditioning costs and transportation premium reported in Table 1 were taken from Dunant et al. (2018b). These cost estimates are based on a set of 30 interviews with architects, structural designers, construction contractors, fabricators, steel stockists and demolition contractors. All costs are reported relative to a reference case: demolishing the unwanted building and recycling the beams, and specifying new beams for the new building.

Lightweight beam. Design codes set performance criteria for beams that make up steel buildings. These codes include safety margins that take into account the risk of failure. Buildings could be designed to use the minimum amount of steel required to meet these performance criteria but instead tend to exceed these criteria. Moynihan and Allwood assessed utilisation of over 12,000 beams and columns in 23 building designs, and found that on average $46 \%$ of steel in beams was surplus to the requirements of design codes. More recent data on 3,600 beams in 30 commercial office and educational projects analysed for the Innovate UK Lightweighting Project found $53 \%$ of steel to be surplus to requirements on average Dunant et al. (2018a). The average cost of the excess steel in 
these buildings was $£ 19$ per beam ranging from $£ 1$ - 419 per beam. Structural engineers may choose to specify more steel than is technically required in order to reduce overall costs by simplifying design and fabrication requirements (a practice referred to as 'rationalisation') or to allow for greater flexibility regarding the final design. Rather than evaluating these costs on a case-by-case basis, structural engineers tend to use rules of thumb to guide their design. For example Gibbons (1995) proposes that sections should be rationalised if the total increase in weight is less than $20 \%$, and, more conservatively Needham (1977) recommends rationalisation up to a $10 \%$ weight increase for "small jobs" and $5 \%$ weight increase for "medium sized jobs". To generate the MAc distribution in Table 2 we assume that designers behave rationally and so infer that at the margin, the cost of excess steel in the building must equal the benefit of rationalisation and the benefit of flexibility to make late alterations to designs.

High strength steel car-body. Innovations in metallurgy have improved the technical properties of steel, meaning that it is possible to make lighter vehicles. Current material choices in the automotive sector are driven by material costs, design vision and the desire to innovate. Given the different properties of materials, substituting one material for another requires full vehicle redesign. The Future Steel Vehicle project by the Automotive arm of the World Steel Association (World Auto Steel, 2011) developed full engineering designs for a lightweight steel-intensive electric vehicle using high strength steels (>500 MPa) and advanced high strength steels (>550 MPa). The project sought to achieve a $35 \%$ body structure weight saving relative to the baseline vehicle. Focussing on manufacturing costs (excluding savings associated with the electric powertrain) the study found an average cost of weight saving of $\$ 7.12 / \mathrm{kg}$. A study by McKinsey (2012) estimates the cost of converting to high strength steels at $€ 3 / \mathrm{kg}$. Converting both the World Auto Steel (2011) and the McKinsey (2012) findings (converted to Pounds Sterling) gives a cost range of $£ 2.49-4.27 / \mathrm{kg}$. The upper limit of this range is given by the World Auto Steel (2011) result regarding changes in part cost. It excludes all powertrain 
cost savings and is likely to be an over-estimate of vehicle costs for a lightweight internal combustion engine car as we would expect some cost savings resulting from a smaller internal combustion engine. Use-phase cost savings associated with the lighter vehicle weight can be included. European legislation takes these savings into account in setting "limit value curves" that define emissions standards as a function of kerbweight. The slope of the limit value curve is $0.0457 \mathrm{gcO}_{2} / \mathrm{km} / \mathrm{kg}$ vehicle (European Environment Agency, 2016). The average car in the uk travels $12,700 \mathrm{~km} /$ year implying a $0.58 \mathrm{kgco}_{2}$ saving per year, per vehicle $\mathrm{kg}$.

Smaller car. There are many different types of cars within the vehicle fleet. Demand for passenger kilometres could be met by small cars within the existing offering. In this section we explore the implications of customers buying the lightest weight steel option currently available within their chosen vehicle segment (e.g. sports car, large family car, city car). In reality consumers are free to choose from a range of vehicles offered by manufacturers depending on their preferences and budget constraints. McKinsey (2012) state that consumers have "limited willingness to pay for weight reduction" suggesting, that kerbweight is not an important determinant of choice. Combining data on car-body mass (from the Euro Car Body Conference), vehicle price (from various sources documented in the Supplementary Information) and on the composition of the UK vehicle fleet from Lansley (2016-01), reveals that downsizing to the lightest steel vehicle within the chosen segment would result in a $12 \%$ reduction in average vehicle mass at an average cost saving of $43 £ / \mathrm{kg}$. Only a fraction of the retail price of a vehicle is associated with the cost of the car body structure. von Thaden et al. (2017) estimate the cost of the body-in-white (BIw) of a mass-produced European compact vehicle at approximately $£ 630$ per vehicle, which represents on average $3 \%$ of vehicle price. Only taking into account this BIW cost share would imply an average cost saving of $1.3 £ / \mathrm{kg}$. This cost saving is consistent with assuming that customers buy vehicles with similar features to their first choice vehicle but a smaller body-in-white, meaning that they only 
realise cost savings associated with the smaller car body.

\subsection{Accounting for interaction between strategies}

The Sankey diagram in Figure 3 is based on data from Serrenho et al. (2016-02) and shows UK steel mass flows focussing on the two key sectors of interest: the construction sector and the automotive sector. It shows which steel mass flows are affected by each of the material efficiency measures. Mapping the measures onto the steel mass Sankey is helpful as it reveals which measures inter-relate. Whether or not adjustments have to be made to account for the interdependencies of these strategies then depends on the specific case.

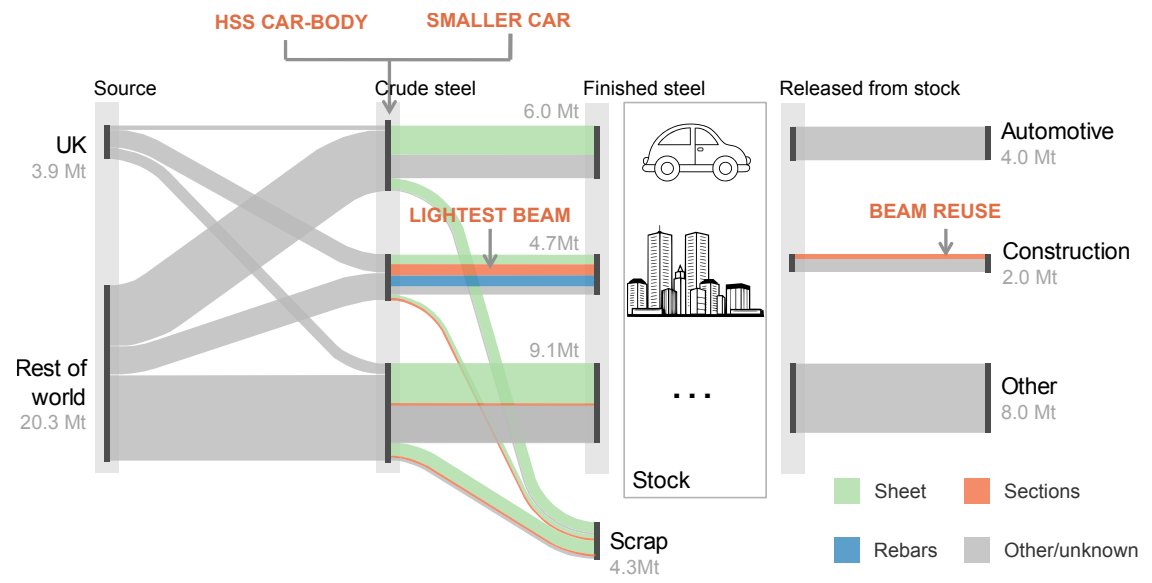

Figure 3: Material efficiency measures mapped onto the the uK steel mass flow sankey.

The lightest beam strategy and the beam reuse strategy both relate to steel section use in the Construction sector. The effect of choosing the lightest beam on the potential for reuse is mediated by the building stock. As the average life of a building is approximately 40 years and as both strategies relate to the use of standardised (as opposed to bespoke) beams, we assume that the two strategies are independent. This means that we are effectively assuming that the specification of beams that are released from stock for reuse can be incorporated into lightweight new building designs. 
In the automotive sector both strategies — choosing the smaller car in the chosen vehicle segment, and specifying a high strength steel car body — relate to the same mass flow. Both strategies depend on the mass of the average car and so the extent to which one strategy can be applied depends on the extent to which the other strategy has been applied. As the costs of the smaller car strategy are less than the cost of specifying high strength steels, we assume that the smaller car strategy is implemented first. Assuming that the smaller car strategy (A) can reduce the average weight of vehicles by $12 \%$ the extent to which the high strength steel strategy (B) can be applied is given by:

$$
\eta_{B \mid A}=1-\left(\gamma_{A} \cdot 0.12\right)
$$

Where $\gamma_{A}$ is the fraction (ranging from 0 to 1 ) that denotes the extent to which the smaller car strategy is applied. If strategy A is not applied at all, strategy B can be applied in full; if strategy A is fully deployed, the gains of strategy B are at most $88 \%$ of its theoretical maximum.

\subsection{Allocating emissions to steel savings}

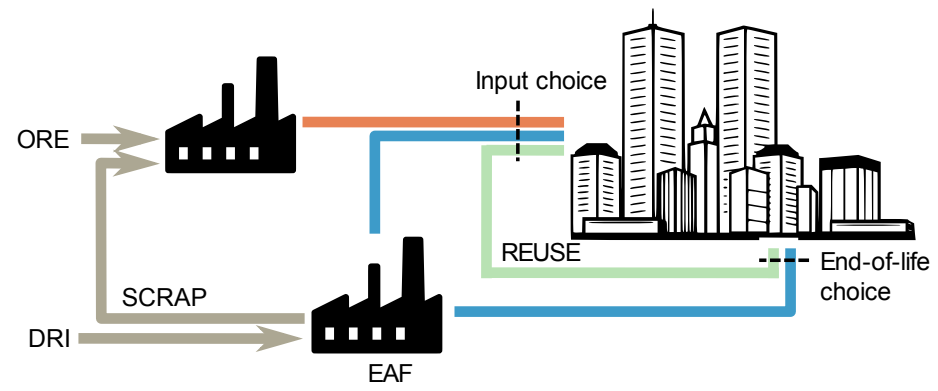

Figure 4: Schematic showing emissions savings associated with reuse of steel sections

256 There are multiple methods that can be used to allocate emissions savings to material efficiency strategies. Like costs, all emissions savings must be measured relative to a reference case that represents what would have happened in the absence of the strategy being applied. By way of example, Figure 4 gives an overview of the steel flows 
associated with replacing a building. The figure shows that when a building is built, steel can be specified from three different sources: primary steel from a blast furnace-basic oxygen furnace(BF-BOF), secondary steel from an electric arc furnace (EAF) or reused steel. At the end of a building's life the choice is more constrained: steel can either be sent for reuse or for recycling. Based on Figure 4, there are different ways to allocate emissions savings to steel reuse in buildings:

- Displaced input method: if the input choice is considered in isolation, increasing the share of reused steel that is specified would displace both steel from iron ore and from recycled scrap

- Displaced scrap method: taking into account the more constrained choice at the end of a building's life, steel reuse can only displace steelmaking from recycled scrap and not steelmaking from iron ore

- Future reuse potential method: steel has the potential to be reused indefinitely therefore the choice to specify steel (rather than other materials such as concrete that has no current viable route for reuse) facilitates future reuse and/or recycling. Methods such as BS EN 15804 (2012) 'Module D' and PAS 2050 (2008) attempt to attribute future potential savings to the current decision.

We use the displaced scrap method for the beam reuse strategy as this method does not rely on uncertain future events and as it takes into account the more constrained choice at the end of a building's life, in line with Sansom and Avery (2014-06) findings that over $90 \%$ of structural steel in buildings is recycled or reused at the end-of-life. The remaining material efficiency strategies examined in this paper relate to input decisions and so we use the displaced input method for these measures. Table 3 gives a summary of the maximum potential material and so emissions savings that can be achieved by each of the measure. In order to allow measures to be compared, all steel savings are calculated in crude steel equivalent units. This means that any measures that occur 
further downstream along the mass sankey diagram in Figure 3 save not only the finished steel in the relevant product but also the yield losses along the supply chain making those products.

Table 3: Measure specific material and Ghg emissions savings. Sources for these values can be found in the sI in the the sections corresponding to each strategy.

\begin{tabular}{llll}
\hline Measure & $\begin{array}{l}\text { Material saving } \\
\text { (Mt cr. steel eq. })\end{array}$ & Emissions displaced & $\begin{array}{l}\text { Emissions saving } \\
\left(\mathrm{Mtco}_{2}\right)\end{array}$ \\
\hline Beam reuse & 0.22 & $100 \%$ EAF & 0.09 \\
Lightest beam & 0.20 & $26 \%$ EAF; $74 \%$ BF-BOF & 0.35 \\
Hss car body & 1.50 & $100 \%$ BF-BOF & 3.40 \\
Smaller car & 0.44 & $100 \%$ BF-BOF & 1.00 \\
\hline
\end{tabular}

\subsection{Building the new MACC}

Figure 5 shows the resulting marginal abatement cost curve for the four material efficiency measures. The top panel shows the graph which only account for emissions savings associated with reduced demand for steel. The bottom panel shows the same MACC when also taking accounting for use-phase costs and emissions. These only affect the automotive sector strategies as the use-phase emissions of buildings is not affected by the proposed changes in frame construction. The marginal abatement cost distributions used as input are given in Table 2 and the greenhouse gas emissions abatement potentials in Table 3. Possible double counting due to the interdependence between the high strength steel car-body measure and the smaller car strategy is corrected for as per Section 3.2. Removing double-counting reduces the abatement potential by $0.38 \mathrm{Mtco}_{2}$ in the steel emissions only case and $0.75 \mathrm{Mtco}_{2}$ when accounting for use phase savings, equivalent to $8 \%$ and $15 \%$ respectively of the total abatement potential.

Figure 6 shows a zoomed in version of three material efficiency MAccs that assume different steel prices ranging from $£ 640$ in the high price scenario to $£ 310$ in the low price scenario (covering for $95 \%$ of cases in the assumed cost distribution). The graph shows that the beam reuse strategy becomes more preferable in the high steel price scenario. This is to be expected as, in accordance with the beam reuse equation in 

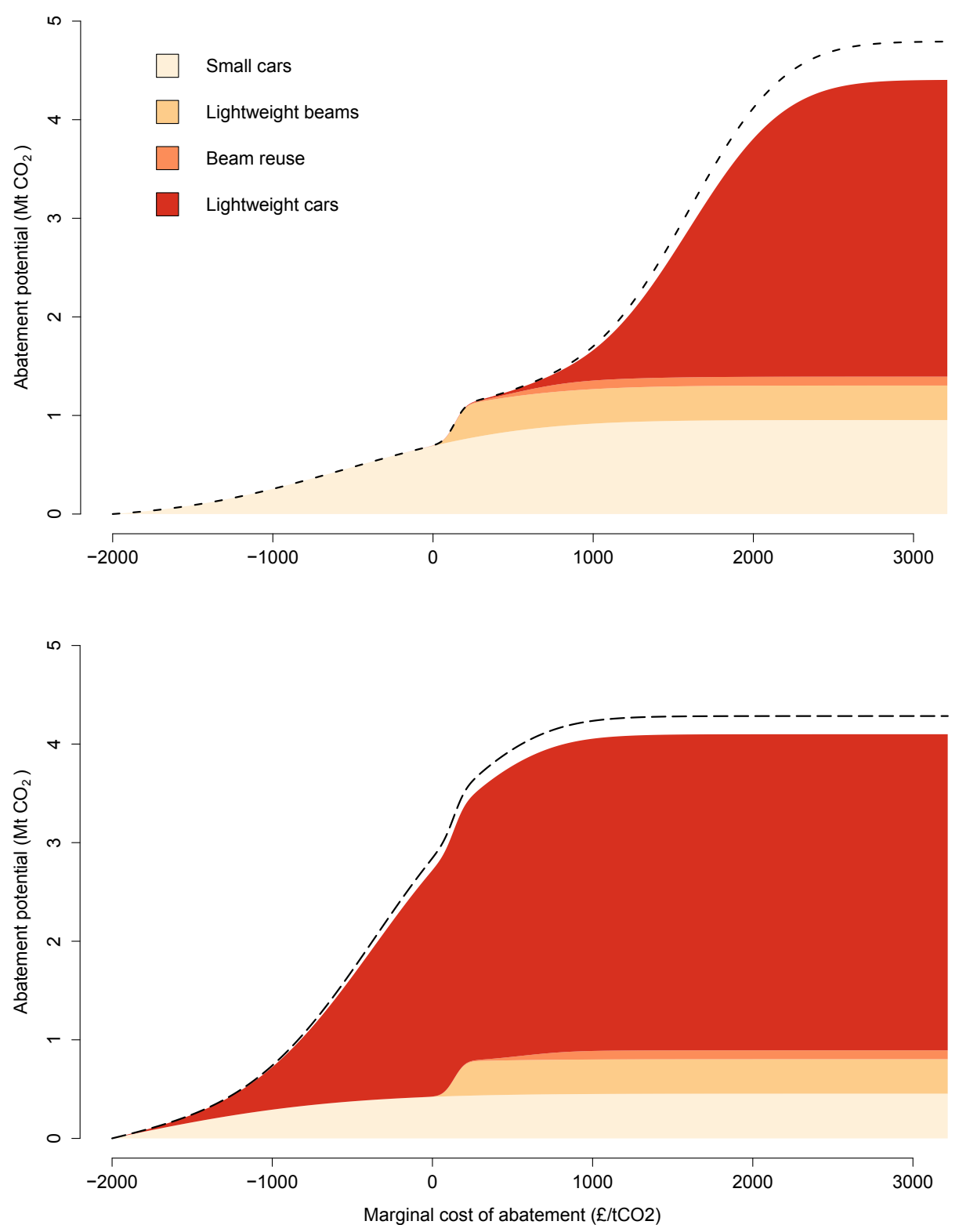

Figure 5: Novel material efficiency MACC accounting for the interdependence between strategies (steel emission savings only on top; including use-phase cost and emissions savings at the bottom). The dashed line indicates the emissions when the interdependency between strategies is not accounted for. Example code to generate these figures is given in 5 .

Table 2, a higher steel price raises the cost of the reference case strategy (specifying

primary steel), increasing the incentives for reuse. Rather counter-intuitively, Figure 6 


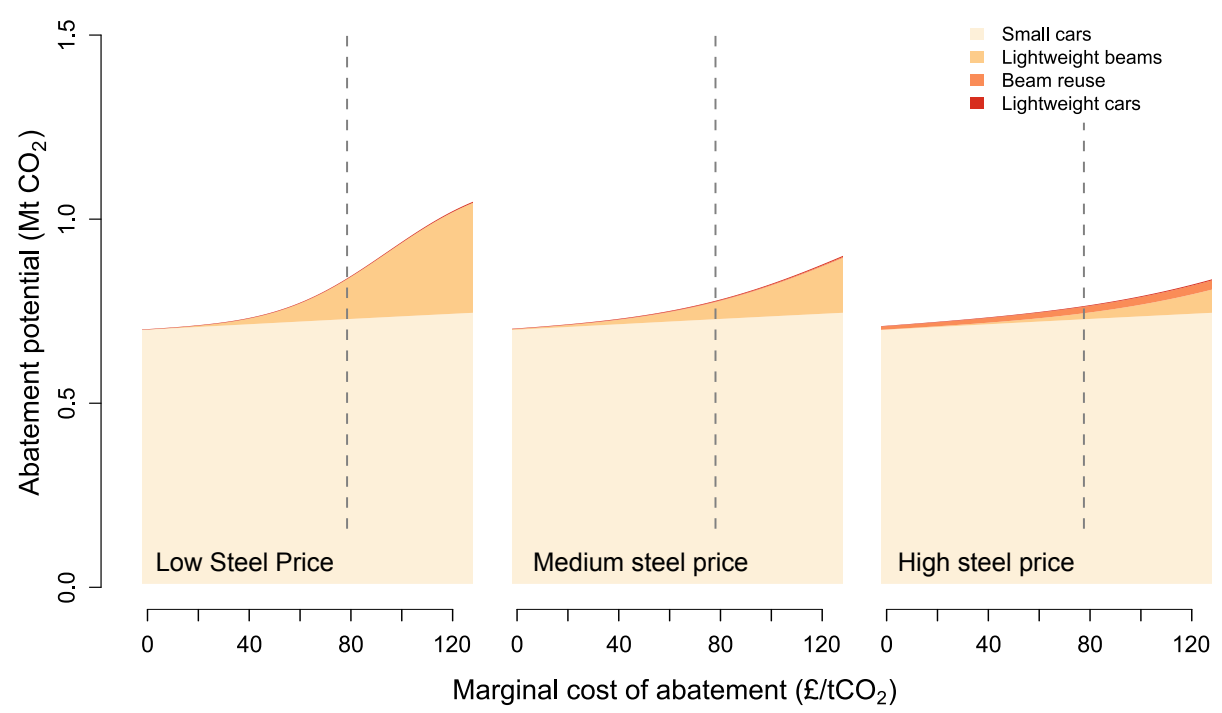

Figure 6: A selection of material efficiency MAccs under different steel price scenarios

shows weaker incentives for the lightweight beam strategy in the high steel price scenario. This is unexpected as a high steel price would increase the incentives for structural engineers to take greater care to avoid over-specifying the amount of steel in beams in excess of the requirements of building codes. This result is caused by the simplicity of the cost model assumed for this strategy. By assuming that the cost of excess steel must equal the benefit of rationalisation and flexibility to react to future design changes we force the unwanted result that the higher the steel price, the higher the benefits of rationalisation and flexibility, and so the lower the incentives to pursue this strategy. This suggests that it is only meaningful to explore price sensitivity if the underlying cost models are sufficiently sophisticated to respond to these price changes sensibly. It is not possible in the scope of this work to develop such a model, which would need to include considerably more information, notably the long-term interest rate, the futures market for steel, as well as predictions for the construction sector.

Rather, such apparently contradictory result highlights where complex behaviour by the actors is the likely underlying cause of inefficient use of materials, and where intervention is likely the hardest. 
3.5. Discussion - material efficiency strategies

Table 4: Measure specific material and Gh emissions savings

\begin{tabular}{llllll}
\hline Measure & Total potential & \multicolumn{4}{c}{ Exploited at $79 £ / \mathrm{tco}_{2}$} \\
& \multicolumn{3}{c}{$\begin{array}{c}\text { Steel only } \\
\text { Steel \& use phase }\end{array}$} \\
& $\left(\mathrm{Mtco}_{2}\right)$ & $\left(\mathrm{Mtco}_{2}\right)$ & $(\%)$ & $\left(\mathrm{Mtco}_{2}\right)$ & $(\%)$ \\
\hline Smaller car & 1.00 & 0.72 & $72 \%$ & 0.42 & $42 \%$ \\
Lightest beam & 0.35 & 0.05 & $14 \%$ & 0.05 & $14 \%$ \\
Beam reuse & 0.09 & 0.002 & $2 \%$ & 0.002 & $2 \%$ \\
Hss car body & 3.40 & 0.002 & $0.05 \%$ & 2.40 & $70 \%$ \\
\hline
\end{tabular}

Table 4 shows the share of abatement that would be considered to be cost effective under the BEIS (2018) $79 £ / \mathrm{tcO}_{2} 2030$ carbon value for policy appraisal. This value is the projected carbon cost under the eu emissions trading scheme which is believed by the BEIs to lead to the mitigation targets being met. The table shows that, when only emissions savings due to reduced demand for steel are taken into account, the cheapest strategy (choosing smaller cars) is largely exploited but the more expensive strategies (steel reuse and high strength steel body in white in this case) are only just starting to come into play. Once use-phase cost and associated emissions savings are taken into account the costs of the two lightweighting strategies in the automotive sector reduced dramatically, increasing incentives for the Hss car body strategy and reducing the efficacy of the smaller car strategy due to the double counting restriction.

The novel material efficiency MAccs in Figure 5 shows that, once double-counting is removed, the four proposed material efficiency measures could save in the order of $4.5 \mathrm{Mt} \mathrm{CO}_{2}$. This represents $35 \%$ of uK direct emissions due to industrial processes DECC (2016). The emissions savings would occur across global supply chains. Cabrera Serrenho et al. (2015) estimate that 20 Mt of steel was used to meet UK demand in 2007, of which 13 Mt was imported. As shown in Table 3, the proposed strategies would reduce steel demand by $2.4 \mathrm{Mt}$, equating to a $12 \%$ reduction in steel demand and associated emissions. The two maccs show a wide range of costs of abatement, with the average cost of abatement for each of the strategies ranging from $-705 £ / \mathrm{tco}_{2}$ for the 
smaller car strategy to $1600 £ / \mathrm{tco}_{2}$ for high strength steel car body strategy in Figure 5

(top) and $-2,135 £ / \mathrm{tcO}_{2}$ for the smaller car strategy to $565 £ / \mathrm{tcO}_{2}$ for the reuse strategy in Figure 5 (bottom).

\section{Discussion}

In this paper we have presented a novel method for building a marginal abatement cost curve (MACC) to take into account material efficiency measures and applied this method to build a marginal abatement cost curve for four material efficiency measures in the UK. In response to calls for greater transparency on MACC assumptions, in the next section (Section 4.2) we set out a standard for documentation for documenting MACC assumptions based on the experience of populating the material efficiency MAcc. We then identify how the novel MACC method proposed here improves on the traditional MACC (Section 4.1).

\subsection{Methodological improvements}

Figure 1 shows the four material efficiency measures in the traditional MACc format. This format has many shortcomings in particular:

- The assumption that there is a single cost for each measure set equal to the average cost for that measure;

- The assumption that measures will be chosen in sequence (from least to highest average cost) rather than in parallel;

- The assumption that measures are independent and so that the abatement potential of individual measures can be summed to reveal the total abatement potential.

The novel material efficiency MAcc in Figure 5 addresses these shortcomings by:

- Depicting a cost distribution for each measure; 
- Identifying parameters that are common across measures, and allowing measures to occur in parallel, where appropriate, for a given cost of carbon under a consistent set of cross-strategy parameters;

- Taking into account the interaction between strategies and so removing double counting of emissions potentials.

Comparing the traditional (Figure 1) and novel (Figure 5) is complicated slightly by the fact that the axes are reversed in the novel MACc as compared to the traditional MACC. The benefit of this modification is that cumulative distribution function (CDF) for different carbon prices can be stacked taking into account the interdependency between strategies. Although this makes it slightly more cumbersome to compare the two MAccs it does not detract from the ease of interpreting the novel MACC when viewed in isolation.

\subsection{Assumptions verification}

The process of estimating the marginal cost of abatement for a range of material efficiency measures is helpful not only for populating the MAcc but also for revealing the types of assumptions that have to be made to estimate a MAcc. What becomes clear is that there can be no definitive MACc, there can only be a particular representation of costs based on a particular set of assumptions. The challenge then is to state the assumptions transparently in order to avoid mis-representation and to be as consistent as possible in the treatment of different strategies in order to allow comparison. Figure 7 gives an overview of the types of assumptions that have to be made in order to populate a material efficiency MAcc. The assumptions are broken into three categories: crossstrategy assumptions, strategy specific assumptions and curve specific assumptions. Any MACc, whether the novel MACc proposed here, or a traditional macc, includes these different types of assumptions although they may not be clearly stated or dealt with consistently.

Identifying cross-strategy assumptions helps to ensure consistency across the emissions abatement strategies considered in the Macc. The key here is to distinguish between 
assumptions that apply across all strategies and must be consistent as opposed to those that apply across all strategies but may differ. For example we would expect the steel price level to be consistent across scenarios (it would be unfair to compare an abatement cost that assumes a low steel price for one strategy to one that assumes a high steel price for another), but allow the emissions intensity of steel to vary across strategies to reflect the range in emissions intensities of current steel plants (as it is possible that steel is sourced from a high emitting source in one case but a lower emitting source in another).

The interdependence of cross-strategy assumptions must also be taken into account: for example the steel scrap price should be modelled as a function of the virgin steel price, rather than modelled independently, as the two prices tend to move together.

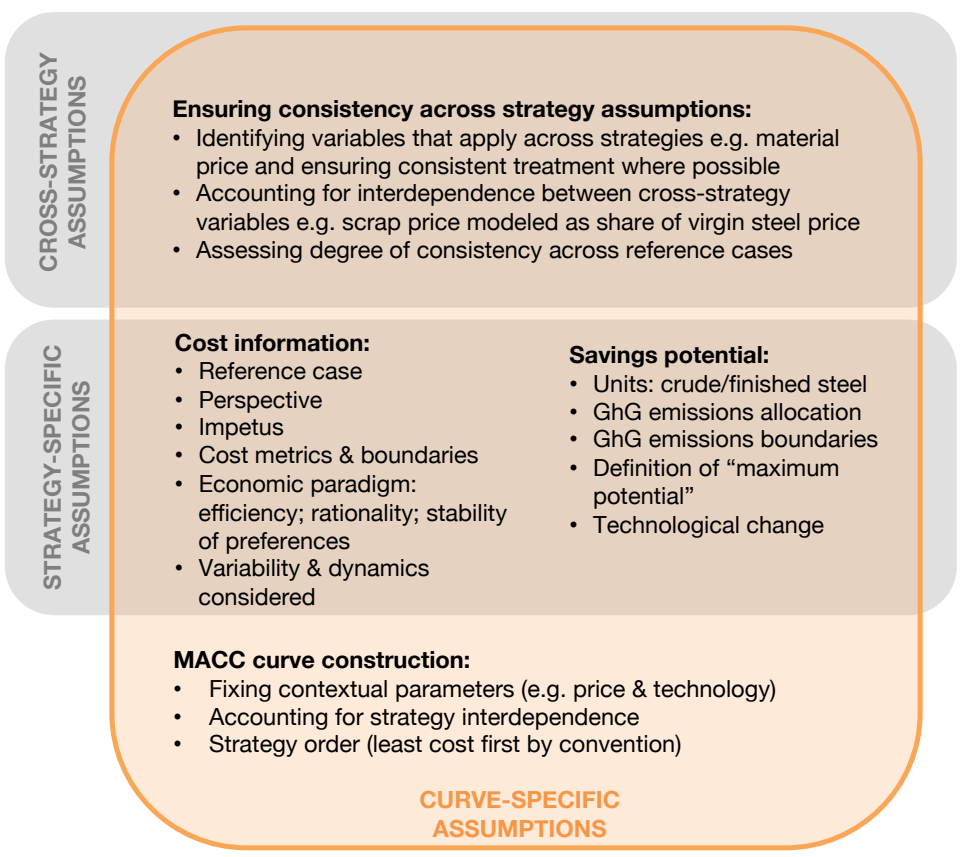

Figure 7: Standard for documentation of the assumptions behind the creation of a MACc for logging material efficiency MAC assumptions

The strategy specific assumptions in Figure 7 break down some of the elements that make up different, at times competing, notions of cost. These are the types of features of costs that researchers should be mindful of when building a MAcc. They relate both 
to the data that are collected to populate the MACC and to the way that these data are interpreted and, as explained in Section 2.2 the interpretation cause as much influence on the magnitude of costs as the underlying data.

Finally, there are a set of curve specific assumptions. These include the crossstrategy and strategy specific assumptions mentioned above, as well as assumptions that relate to the construction of the particular curve itself. In order to construct a single cost curve (as opposed to a cost fan) all contextual parameters (such as steel prices in this case) must be held constant. The curve can then be constructed for the given set of contextual parameters by stacking the CDF for cost assumed for each strategy. Assumptions regarding the nature of the interdependence between strategies are then required in order to remove any double-counting of abatement potential from the curve. Given the nature of the curve, the convention is to assume that lower cost measures are pursued first.

\section{Example R code}

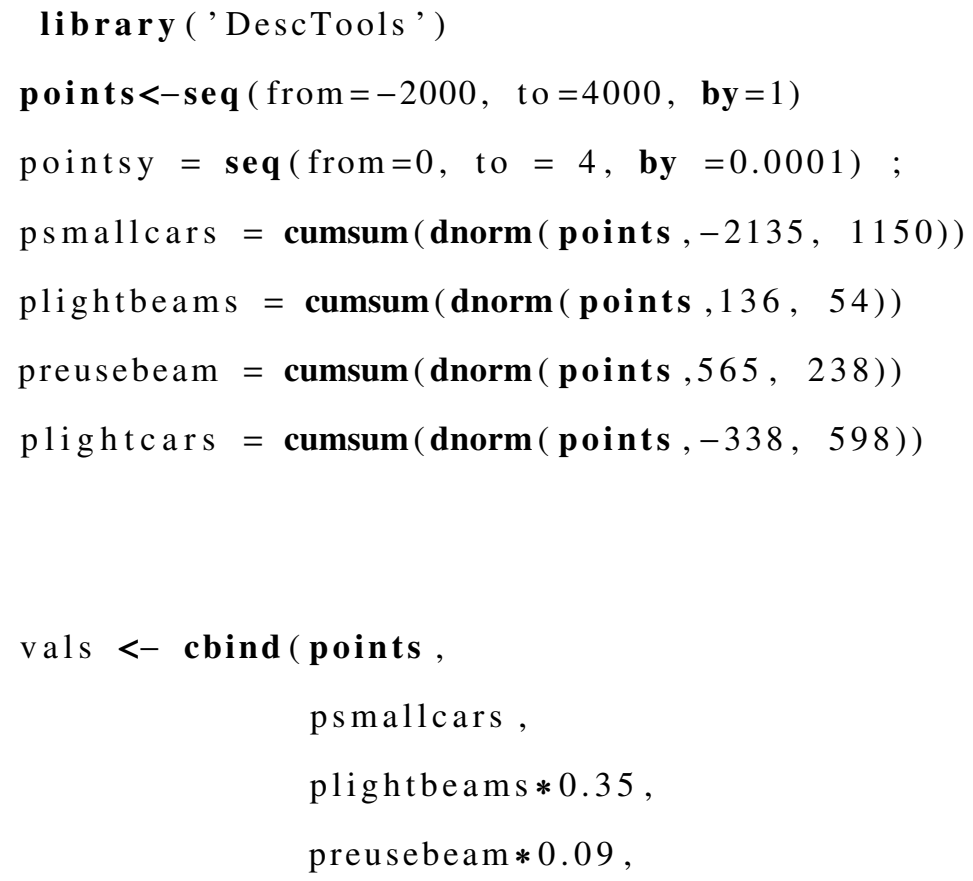




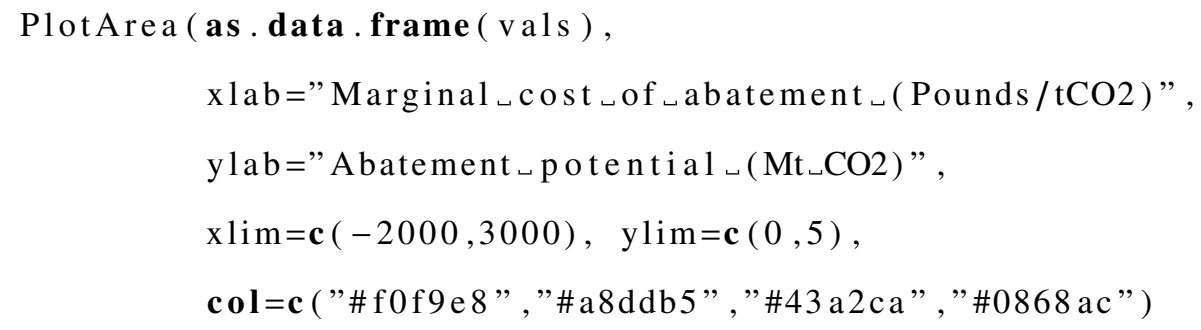

Maccs have been used to guide policy. They provide context for possible abatement measures, and provide a simple mean to estimate economic impacts and pathways. Unfortunately, because they do not account for the variability and uncertainty of the estimations, nor the interactions between the measures considered, they are a poor guide. The new type of curve proposed here should give a more robust answer to the question 'how much carbon can be abated for what marginal price?'

In the original McKinsey curve, as in our example, some of the measures have a negative cost. This does not mean that the abatement measures should have already been implemented, or that the assumptions are (necessarily) false, Rather, this may reflect uncertainties or the relative novelty of the possible measures: this curve does 
not represent an equilibrium, but rather the driving potential for change on a range of carbon costs.

The new approach requires more data than the traditional one: material flow maps are required to robustly assess the interaction between the abatement measures. These data can be time-consuming to acquire and be somewhat uncertain. Nonetheless they are central to calculating the interactions between measures, and we believe this to be necessary for the curve to be a meaningful predictive tool. Importantly, collecting them informs on the uncertainty and variability of the underlying data, both aspects reflected in the proposed methodology, and notably absent in traditional Maccs.

\section{Conclusions}

This paper has set out a novel method for building a marginal abatement cost curve (MACC). Applying this method to four material efficiency measures in the uK shows that these strategies could deliver significant abatement potential at costs that become competitive once use-phase savings are taken into account. The process of populating the MACC revealed the types of assumptions that have to be made in order to populate a MAcc. These assumptions were summarised into a structured assumptions guide to aid MACc transparency. This novel approach allowed us to lay out an abatement strategy focused on material use, accounting for the uncertainty and variability of the underlying data.

\section{Acknowledgements}

This work was supported by EPSRc Material demand reduction: NMZL/112, RG82144, EPSRC reference: EP/N02351X/1.

\section{References}

Allwood, J.M., Ashby, M.F., Gutowski, T.G., Worrell, E., 2011. Material efficiency: A white paper. Resources, Conservation and Recycling 55, 362-381. doi:10.1016/j.resconrec.2010.11.002. 
BEIS, 2018. Updated short-term traded carbon values.

BS EN 15804, 2012. 15804: 2012: Sustainability of construction works. environmental product declarations. core rules for the product category of construction products. Brussels: CEN .

Cabrera Serrenho, A., Sobral-Mourao, Z., Norman, J., Cullen, J., Allwood, J., 2015. Options to supply the UK steel demand and meet the $\mathrm{CO} 2$ emissions targets, in: The ISIE (International Society for Industrial Ecology) Conference 2015-Taking Stock of Industrial Ecology, University of Bath.

Clarke, L., Jiang, K., 2014. Chapter 6: Assessing transformation pathways, in: Climate Change 2014: Mitigation of Climate Change. Contribution of Working Group III to the Fifth Assessment Report of the Intergovernmental Panel on Climate Change. Cambridge University Press.

DECC, 2009. Carbon valuation in UK policy appraisal: A revised approach.

DECC, 2016. 2014 UK Greenhouse Gas Emissions, Final Figures.

Distelkamp, M., Meyer, M., 2014. Report about resource reduction cost curves for material consumption in different MS and sectors.

Dunant, C.F., Drewniok, M., Eleftheriadis, S., Cullen, J.M., Allwood, J.M., 2018 a. Regularity and optimisation practice in steel structural frames in real design cases. Resources, Conservation \& Recycling 134, 294-302.

Dunant, C.F., Drewniok, M.P., Sansom, M., Corbey, S., Cullen, J.M., Allwood, J.M., 2018b. Options to make steel reuse profitable: An analysis of cost and risk distribution across the uk construction value chain. Journal of Cleaner Production 183, 102-111.

Ekins, P., Kesicki, F., Smith, A.Z., 2011. Marginal abatement cost curves: A call for caution. 
Eory, V., MacLeod, M., Topp, C.F.E., Rees, R.M., Webb, J., McVittie, A., Wall, E., Borthwick, F., Watson, C., Waterhouse, A., 2015. Review and update the uk agriculture marginal abatement cost curve to assess the greenhouse gas abatement potential for the 5 th carbon budget period and to 2050 .

European Environment Agency, 2016. Monitoring CO2 emissions from new passenger cars and vans in 2015 .

Gibbons, C., 1995. Economic steelwork design. The Structural Engineer 73, 250-253.

Horton, P.M., Allwood, J.M., 2017. Yield improvement opportunities for manufacturing automotive sheet metal components. Journal of Materials Processing Technology 249, 78-88. doi:10.1016/j.jmatprotec.2017.05.037.

Kesicki, F., Strachan, N., 2011-12. Marginal abatement cost (MAC) curves: Confronting theory and practice. Environmental Science \& Policy 14, 1195-1204. doi:10.1016/j.envsci.2011.08.004.

Lansley, G., 2016-01. Cars and socio-economics: Understanding neighbourhood variations in car characteristics from administrative data. Regional Studies, Regional Science 3, 264-285. doi:10.1080/21681376.2016.1177466.

McKinsey, 2009. Pathways to a low-carbon economy: Version 2 of the global greenhouse gas abatement cost curve.

McKinsey, 2012. Lightweight, heavy impact.

Moynihan, M.C., Allwood, J.M., 2014. Utilization of structural steel in buildings. Proceedings of the Royal Society A: Mathematical, Physical and Engineering Sciences 470, 20140170-20140170. doi:10.1098/rspa.2014.0170.

Needham, F., 1977. The economics of steelwork design. The Structural Engineer 55, $367-373$. 
PAS 2050, B., 2008. Guide to PAS 2050: How to assess the carbon footprint of goods and services.

Sansom, M., Avery, N., 2014-06. Briefing: Reuse and recycling rates of UK steel demolition arisings. Proceedings of the Institution of Civil Engineers - Engineering Sustainability 167, 89-94. doi:10.1680/ensu.13.00026.

Serrenho, A.C., Mourao, Z., Norman, J., Cullen, J., Allwood, J., 2016-02. The influence of UK emissions reduction targets on the emissions of the global steel industry. Resources, Conservation and Recycling 107, 174-184. doi:10.1016/j.resconrec.2016.01.001.

Skelton, A.C.H., Allwood, J.M., 2017-06-13. The carbon price: A toothless tool for material efficiency? Philosophical Transactions of the Royal Society A: Mathematical, Physical and Engineering Sciences 375, 20160374. doi:10.1098/rsta.2016.0374.

von Thaden, G., Mogge, F., Riederle, S., 2017. Automotive metal components for car bodies and chassis - Global market study.

World Auto Steel, 2011. Future steel vehicle - nature's way to mobility. Overview report. 